\title{
ON HARMONICITY IN A DISC AND $n$-HARMONICITY
}

\author{
JAESUNG LEE
}

\begin{abstract}
Let $\tau \neq \delta_{0}$ be either a power bounded radial measure with compact support on the unit disc $D$ with $\tau(D)=1$ such that there is a $\delta>0$ so that $|\hat{\tau}(s)| \neq 1$ for every $s \in \Sigma(\delta) \backslash\{0,1\}$, or just a radial probability measure on $D$. Here, we provide a decomposition of the set $\mathbf{X}=\left\{h \in L^{\infty}(D) \mid \lim _{n \rightarrow \infty} h * \tau^{n}\right.$ exists $\}$. Let $\tau_{1}, \ldots, \tau_{n}$ be measures on $D$ with above mentioned properties. Here, we prove that if $f \in L^{\infty}\left(D^{n}\right)$ satisfies an invariant volume mean value property with respect to $\tau_{1}, \ldots, \tau_{n}$, then $f$ is $n$-harmonic.
\end{abstract}

\section{Introduction}

Let $D$ be the open unit disc of $\mathbb{C}, \nu$ be the Lebesgue measure on $\mathbb{C}$ normalized to $\nu(D)=1$ and let $\mu$ be the conformally invariant measure on $D$ defined by $d \mu(z)=\left(1-|z|^{2}\right)^{-2} d \nu(z)$, which satisfies

$$
\int_{D} u d \mu=\int_{D} u \circ \varphi d \mu
$$

for every $u \in L^{1}(\mu)$ and for every $\varphi \in \operatorname{Aut}(D)$. And then let us denote $L_{R}^{p}(\mu)$ to be the subspace of $L^{p}(\mu)$ which consists of radial functions.

It is known that (see [3], [4]) $L_{R}^{1}(\mu)$ is a commutative Banach algebra under the convolution

$$
(u * v)(z)=\int_{D} u\left(\varphi_{z}(x)\right) v(x) d \mu(x), \text { where } \varphi_{z}(x)=\frac{z-x}{1-\bar{z} x} .
$$

Likewise if $\tau$ is a radial measure with $\tau(D)=1$, we define the convolution $u * \tau$ on $D$ by

$$
(u * \tau)(z)=\int_{D} u\left(\varphi_{z}(x)\right) d \tau(x)
$$

Received March 2, 2009.

2000 Mathematics Subject Classification. Primary 31B30; Secondary 47B38.

Key words and phrases. mean value property, harmonicity, $n$-harmonicity, convolution, spectrum.

This work was supported by the 2008 Special Research Grant of Sogang University. 
For $f \in L_{R}^{1}(\mu)$, we define its Gelfand transform by

$$
\hat{f}(s)=\int_{D} f(z)\left(\frac{1-|z|^{2}}{|1-z|^{2}}\right)^{s} d \mu(z), \quad 0 \leq \Re s \leq 1 .
$$

Likewise if $\tau$ is a radial measure with $\tau(D)=1$, its Gelfand transform is defined by

$$
\hat{\tau}(s)=\int_{D}\left(\frac{1-|z|^{2}}{|1-z|^{2}}\right)^{s} d \tau(z) .
$$

We know that if $u \in L^{1}(D, \tau)$ is harmonic, then $u \circ \varphi$ is also harmonic for every $\varphi \in \operatorname{Aut}(D)$.

Thus $u \circ \varphi$ satisfies a volume mean value property;

$$
u(\varphi(0))=\int_{D} u \circ \varphi d \tau \text { for every } \varphi \in \operatorname{Aut}(D),
$$

which is equivalent to $u * \tau=u$.

One of the main results of [1] is that if $\nu$ is a normalized Lebesgue measure and $u \in L^{1}(D, \nu)$ satisfies $u * \nu=u$, then $u$ is harmonic. Much earlier, Furstenberg [5], [6] proved that if $m$ is a radial probability measure on $D$ and $u \in L^{\infty}(D)$ satisfies $u * m=u$, then $u$ is harmonic. Indeed, his result says much more is true: On any dimensional symmetric domain, a bounded function which satisfies a certain convolution type of mean value property is harmonic with respect to the intrinsic metric. For example, applying Furstenberg's result to the polydisc $D^{n}$, we get that if $m_{1}, \ldots, m_{n}$ are radial probability measures on $D$, and if $f \in L^{\infty}\left(D^{n}\right)$ satisfies

$$
f(\psi(0, \ldots, 0))=\int_{D} \cdots \int_{D} f \circ \psi d m_{1} \cdots d m_{n} \quad \text { for every } \psi \in \operatorname{Aut}\left(D^{n}\right),
$$

then $f$ is $n$-harmonic (It means $\Delta_{1} f=\cdots=\Delta_{n} f=0$ ).

In 1992, Benyamini and Weit [3] introduced another type of non-positive measure on $D$ and got results analogous to those of Furstenberg. Here we state two theorems of [3].

Theorem 1.1 ([3, Theorems 2.1 and 2.3]). Let $\tau\left(\tau \neq \delta_{0}\right)$ be a power bounded radial measure with compact support on $D$ with $\tau(D)=1$, and there is a $\delta>0$ so that $|\hat{\tau}(s)| \neq 1$ for every $s \in \Sigma(\delta) \backslash\{0,1\}$, where $\Sigma(\delta)$ is the strip $-\delta<\Re s<1+\delta$. Or let $\tau$ be a radial probability measure on $D$. If $u \in L_{R}^{1}(\mu)$ satisfies $\int_{D} u d \mu=0$, then $u * \tau^{n} \rightarrow 0$ in the norm of $L^{1}(\mu)$.

Theorem 1.2 ([3, Theorem 3.1]). Let $\tau\left(\tau \neq \delta_{0}\right)$ be a power bounded radial measure with compact support on $D$ with $\tau(D)=1$, and there is a $\delta>0$ so that $|\hat{\tau}(s)| \neq 1$ for every $s \in \Sigma(\delta) \backslash\{0,1\}$, where $\Sigma(\delta)$ is the strip $-\delta<\Re s<1+\delta$. Or let $\tau$ be a radial probability measure on D. If $v \in L^{\infty}(D)$ satisfies $v * \tau=v$, then $v$ is harmonic.

This paper, in Theorem 1.3, we use the results of Benyamini and Weit (Theorems 1.1 and 1.2) to investigate the existence of the limit of $h * \tau^{n}$ for 
$h \in L^{\infty}(D)$. Indeed, we decompose the space $\mathbf{X}=\left\{h \in L^{\infty}(D) \mid \lim _{n \rightarrow \infty} h *\right.$ $\tau^{n}$ exists $\}$ as a direct sum of the space of bounded harmonic functions and the closure of the space $A=\left\{h-h * \tau \mid h \in L^{\infty}(D)\right\}$. And then, in Theorem 1.4, we prove that Theorem 1.2 can extend to the $n$ dimensional polydisc $D^{n}$. Indeed, one can easily see that if $f$ is an integrable $n$-harmonic function on $D^{n}$ and $\tau_{1}, \ldots, \tau_{n}$ are radial measures on $D$ with $\tau_{1}(D)=\cdots=\tau_{n}(D)=1\left(\tau_{1}, \ldots, \tau_{n}\right.$ need not be positive measures.), then for every $z_{1}, \ldots, z_{n} \in D$, we have

$$
f\left(z_{1}, \ldots, z_{n}\right)=\int_{D} \cdots \int_{D} f\left(\varphi_{z_{1}}\left(x_{1}\right), \cdots, \varphi_{z_{n}}\left(x_{n}\right)\right) d \tau_{1}\left(x_{1}\right) \cdots d \tau_{n}\left(x_{n}\right) .
$$

Recently, the author [8] proved that in case $n \geq 2, f \in L^{p}\left(D^{n}, \tau_{1} \times \cdots \times \tau_{n}\right)$ for $1 \leq p<\infty$, satisfying (1.2) does not imply $n$-harmonicity even if $\tau_{1}, \ldots, \tau_{n}$ are radial probability measures. This paper, Theorem 1.4 asserts that, in case $f \in$ $L^{\infty}\left(D^{n}\right)$, if $\tau_{1}, \ldots, \tau_{n}$ satisfy the conditions of the above mentioned Benyamini and Weit's theorems, then satisfying (1.2) implies $n$-harmonicity of $f$. Here are our main results.

Theorem 1.3. Let $\tau \neq \delta_{0}$ be a power bounded radial measure with compact support on $D$ with $\tau(D)=1$, and there is a $\delta>0$ so that $|\hat{\tau}(s)| \neq 1$ for every $s \in \Sigma(\delta) \backslash\{0,1\}$. Or let $\tau$ be a radial probability measure on D. Suppose we denote $\mathbf{X}=\left\{h \in L^{\infty}(D) \mid \lim _{n \rightarrow \infty} h * \tau^{n}\right.$ exists $\}, H$ the set of all bounded harmonic functions in $D$ and $A=\left\{h-h * \tau \mid h \in L^{\infty}(D)\right\}$. Then $\mathbf{X}$ can be decomposed as $\mathbf{X}=H \oplus \bar{A}$, where $\bar{A}$ is the closure of $A$. Also, if there is $C>0$ such that $|\tau| \leq C \mu$, then $\mathbf{X}$ is a proper subset of $L^{\infty}(D)$.

Theorem 1.4. Let $\tau_{1}, \ldots, \tau_{n}$ be radial measures on $D$ with compact support and $\tau_{i}(D)=1, \tau_{i} \neq \delta_{0}$ for $1 \leq i \leq n$. And suppose, for each $1 \leq i \leq n$ there is $a \delta_{i}>0$ so that $\left|\hat{\tau}_{i}(s)\right| \neq 1$ for every $s \in \Sigma\left(\delta_{i}\right) \backslash\{0,1\}$. Or let $\tau_{1}, \ldots, \tau_{n}\left(\tau_{i} \neq \delta_{0}\right.$ for $1 \leq i \leq n)$ be radial probability measures on $D$. If $f \in L^{\infty}\left(D^{n}\right)$ satisfies

$$
f\left(z_{1}, \ldots, z_{n}\right)=\int_{D} \cdots \int_{D} f\left(\varphi_{z_{1}}\left(x_{1}\right), \ldots, \varphi_{z_{n}}\left(x_{n}\right)\right) d \tau_{1}\left(x_{1}\right) \cdots d \tau_{n}\left(x_{n}\right)
$$

for every $z_{1}, \ldots, z_{n} \in D$, then $f$ is n-harmonic.

We provide the proof of Theorem 1.3 in Section 2 and proof of Theorem 1.4 in Section 3.

\section{Iterates of convolutions}

Now we will prove Theorem 1.3 and then, in Proposition 2.1, we will express the subspace $A=\left\{h-h * \tau \mid h \in L^{\infty}(D)\right\}$ of $L^{\infty}(D)$ in Theorem 1.3 in terms of iterates of convolutions. In the proof of Theorem 1.3, we will do the case when $\tau \neq \delta_{0}$ is a power bounded radial measure with compact support on $D$ with $\tau(D)=1$, and there is a $\delta>0$ so that $|\hat{\tau}(s)| \neq 1$ for every $s \in \Sigma(\delta) \backslash\{0,1\}$. In the case when $\tau$ is a radial probability measure, the proof is identical. 
Proof of Theorem 1.3. First, we'll show that $\mathbf{X} \subset H+\bar{A}$.

For an $h \in \mathbf{X}$, let $P h \in L^{\infty}(D)$ satisfy $\lim _{n \rightarrow \infty}\left\|h * \tau^{n}-P h\right\|_{\infty}=0$. Then $P h$ is continuous on $D$ and the convergence is also pointwise almost everywhere, thus by the dominated convergence theorem, we have

$$
(P h) * \tau=P(h * \tau)=\lim _{n \rightarrow \infty} h * \tau^{n+1}=P h .
$$

Thus by Theorem 1.2, $P h$ is harmonic in $D$. Now we denote $h_{1}=P h$ and $h_{2}=h-P h$ and then we get $h=h_{1}+h_{2}$. Now we'll prove that $h_{2} \in \bar{A}$.

For $g \in L^{1}(\nu)$ we define $B_{\tau} g=g * \tau$. Then we see that $A=\left(I-B_{\tau}\right) L^{\infty}(D)$. Now let $d \in L^{\infty}(D)^{*}$ satisfy $\left\langle d, g-B_{\tau} g\right\rangle=0$ for every $g \in L^{\infty}(D)$, then we get $\left\langle B_{\tau}^{*} d-d, g\right\rangle=0$ for every $g \in L^{\infty}(D)$. This means that $B_{\tau}^{*} d=d$. Hence we have

$$
\langle d, h-P h\rangle=\left\langle\left(B_{\tau}^{*}\right)^{k} d, h-P h\right\rangle=\left\langle d, B_{\tau}^{k}(h-P h)\right\rangle \text { for all } k .
$$

But from the definition of the operator $P$, we see that

$$
\lim _{k \rightarrow \infty}\left\|B_{\tau}^{k}(h-P h)\right\|_{\infty}=0 .
$$

Thus, by taking the limit $k \rightarrow \infty$ in $(2.1)$, we get $\langle d, h-P h\rangle=0$. Hence by the Hahn-Banach theorem, $h_{2}=h-P h$ is in the closure of $\left(I-B_{\tau}\right) L^{\infty}(D)$ and this proves that $\mathbf{X} \subset H+\bar{A}$.

On the other hand, for $\psi \in \operatorname{Aut}(D)$ and $z \in D, \varphi_{\psi(z)} \circ \psi \circ \varphi_{z}$ takes 0 to 0 thus is $e^{i \theta}$ for some $\theta$. Hence by rotation-invariance of $\tau$,

$$
B_{\tau}(g \circ \psi)(z)=\int_{D} g\left(\left(\psi\left(\varphi_{z}(x)\right)\right) d \tau(x)=\int_{D} g\left(\varphi_{\psi(z)} e^{i \theta} x\right) d \tau(x)=\left(B_{\tau} g\right)(\psi(z)) .\right.
$$

Also if $u \in L_{R}^{1}(\mu)$ and $v \in L_{R}^{\infty}(D)$, then we get

$$
\int_{D} u \cdot\left(B_{\tau} v\right) d \mu=(u * v * \tau)(0)=(u * \tau * v)(0)=\int_{D}\left(B_{\tau} u\right) \cdot v d \mu .
$$

This means that the operator $B_{\tau}$ on $L_{R}^{\infty}(D)$ is the adjoint of $B_{\tau}$ on $L_{R}^{1}(\mu)$. And, since $L_{R}^{\infty}(D)$ is the dual space of $L_{R}^{1}(\mu)$, we see that the spectrum of $B_{\tau}$ on $L_{R}^{\infty}(D)$ is the same as the spectrum of $B_{\tau}$ on $L_{R}^{1}(\mu)$. Now let $\lambda$ be in the spectrum of $B_{\tau}$ on $L^{\infty}(D)$, then there exists a sequence $\left\{g_{k}\right\}$ in $L^{\infty}\left(B_{n}\right)$ with $\left\|g_{k}\right\|_{\infty}=1$ satisfying $\lim _{k \rightarrow \infty}\left\|B_{\tau} g_{k}-\lambda g_{k}\right\|_{\infty}=0$.

Let $\phi_{k} \in \operatorname{Aut}(D)$ satisfy $\left\|R\left(g_{k} \circ \phi_{k}\right)\right\|_{\infty}=1$ where $R g(z)=\frac{1}{2 \pi} \int_{0}^{2 \pi} g\left(z e^{i \theta}\right) d \theta$ denotes the radialization of $g$. Since $B_{\tau}$ and $R$ are bounded on $L^{\infty}\left(B_{n}\right)$, we have

$$
\begin{aligned}
\left\|B_{\tau}\left(R\left(g_{k} \circ \phi_{k}\right)\right)-\lambda R\left(g_{k} \circ \phi_{k}\right)\right\|_{\infty} & =\left\|R\left(B_{\tau}\left(g_{k} \circ \phi_{k}\right)\right)-R\left(\lambda g_{k} \circ \phi_{k}\right)\right\|_{\infty} \\
& \leq\left\|B_{\tau}\left(g_{k} \circ \phi_{k}\right)-\lambda g_{k} \circ \phi_{k}\right\|_{\infty} \\
& =\left\|\left(B_{\tau} g_{k}\right) \circ \phi_{k}-\lambda g_{k} \circ \phi_{k}\right\|_{\infty} \\
& =\left\|B_{\tau} g_{k}-\lambda g_{k}\right\|_{\infty} \rightarrow 0 \text { as } k \rightarrow \infty .
\end{aligned}
$$

Hence $\lambda$ is in the spectrum of $B_{\tau}$ on $L_{R}^{\infty}(D)$. 
In the proof of Theorem 2.3 of [3], it is shown that the spectrum of $B_{\tau}$, as an operator on $L_{R}^{1}(\mu)$, intersects the unit circle at most at the single point 1 . Hence, by the above argument, the spectrum of $B_{\tau}$ on $L^{\infty}(D)$ also intersects the unit circle at most at the single point 1. By the theorem of Katznelson and Tzafriri [7, Theorem 1], we see that

$$
\lim _{k \rightarrow \infty}\left\|B_{\tau}^{k}\left(I-B_{\tau}\right)\right\|=0 \text { on } L^{\infty}(D) .
$$

By (2.2) we see that if $h \in \bar{A}$, then $h * \tau^{n} \rightarrow 0$ in $L^{\infty}(D)$ and thus $H \cap \bar{A}=\{0\}$. Therefore, we get $\mathbf{X}=H \oplus \bar{A}$ and to complete the proof, it remains to show that $\mathbf{X}$ is a proper subset of $L^{\infty}(D)$ when we assume that there is $C>0$ such that $|\tau| \leq C \mu$. Theorem 6.1 of [2] deals with a similar case.

Suppose we assume that $\mathbf{X}=L^{\infty}(D)$. Then $\lim _{n \rightarrow \infty} h * \tau^{n}$ exists for every $h \in L^{\infty}(D)$.

Now we choose $u \in L_{R}^{1}(\mu)$ with $\int_{D} u d \mu \neq 0$. Then for every $\ell \in L_{R}^{\infty}(D)$,

$$
\lim _{n \rightarrow \infty} \int_{D} \ell \cdot\left(u * \tau^{n}\right) d \mu=\lim _{n \rightarrow \infty} \int_{D} u \cdot\left(\ell * \tau^{n}\right) d \mu \text { exists. }
$$

Since $L_{R}^{1}(\mu)$ is weak complete, $u * \tau^{n}$ converges weakly to some $v \in L_{R}^{1}(\mu)$. And then $u * \tau^{n+1}$ converges to $v * \tau$, which implies that $v * \tau=v$. For each $z \in D$, we have

$$
|v(z)|=|(v * \tau)(z)| \leq \int_{D}\left|v \circ \varphi_{z}\right| d|\tau| \leq C \int_{D}\left|v \circ \varphi_{z}\right| d \mu=C \int_{D}|v| d \mu=\|v\|_{L^{1}(\mu)} .
$$

Thus $v$ is bounded and by Furstenberg's theorem, $v$ is harmonic in $D$. Since a constant is the only radial harmonic function, and since 0 is the only constant that belongs to $L^{1}(\mu)$, we conclude that $v$ is the constant zero. Now putting $\ell=1$ to the integral in $(2.3)$, we get

$$
\int_{D} u d \mu=\int_{D} u \cdot\left(1 * \tau^{n}\right) d \mu=\int_{D} u * \tau^{n} d \mu
$$

which tends to 0 as $n \rightarrow \infty$ to conclude that $\int_{D} u d \mu=0$ contradicting our assumption.

Therefore, $\mathbf{X}$ is a proper subset of $L^{\infty}(D)$ when we assume that there is $C>0$ such that $|\tau| \leq C \mu$ and this completes the proof of the theorem.

In the proof of Theorem 1.3, we've shown that

$$
\bar{A}=\left\{h \in L^{\infty}(D) \mid \lim _{n \rightarrow \infty} h * \tau^{n}=0\right\} .
$$

Now in the following proposition, we will show that the space $A$ can be expressed in terms of iterates of convolutions.

Proposition 2.1. With the same assumptions and notations as Theorem 1.3, we have

$$
A=\left\{h \in L^{\infty}(D) \mid \limsup _{n \rightarrow \infty}\left\|\sum_{0}^{n} h * \tau^{k}\right\|_{\infty}<\infty\right\}
$$


Proof. Let $f=h-h * \tau$ for some $h \in L^{\infty}(D)$ and let $K=\sup \left\|\tau^{n}\right\|$. Then $\left\|\sum_{0}^{n} f * \tau^{k}\right\|_{\infty}=\left\|h-h * \tau^{n+1}\right\|_{\infty} \leq(1+K)\|h\|_{\infty}$. Thus we can see that

$$
A \subset\left\{h \in L^{\infty}(D) \mid \limsup _{n \rightarrow \infty}\left\|\sum_{0}^{n} h * \tau^{k}\right\|_{\infty}<\infty\right\} .
$$

On the other hand, pick $h \in L^{\infty}(D)$ such that $\limsup \left\|\sum_{0}^{n} h * \tau^{k}\right\|_{\infty}=M<$ $\infty$.

Now if we denote $h_{k}=\sum_{j=0}^{k} h * \tau^{j}$, then $h_{k}-h_{k} * \tau=h-h * \tau^{k+1}$. Hence if we let $H_{n}=\frac{1}{n+1} \sum_{k=0}^{n} h_{k}$, then we get $\left\|F_{n}\right\|_{\infty} \leq M$ and we also have

$$
\begin{aligned}
H_{n}-H_{n} * \tau & =\frac{1}{n+1} \sum_{k=0}^{n}\left(h_{k}-h_{k} * \tau\right) \\
& =\frac{1}{n+1} \sum_{k=0}^{n}\left(h-h * \tau^{k+1}\right) \\
& =h-\frac{1}{n+1} \sum_{k=0}^{n} h * \tau^{k+1} .
\end{aligned}
$$

Hence

$$
\left\|H_{n}-H_{n} * \tau-h\right\|_{\infty} \leq \frac{1}{n+1} M \rightarrow 0 .
$$

But a norm bounded sequence $H_{n}$ has a subsequence $H_{n_{j}}$ that converges weak* to some $g \in L^{\infty}(D)$ and as in the proof of Theorem 1.3, the operator $I-B_{\tau}$ is self-adjoint in $L^{1}(\mu)$, which makes $\left(I-B_{\tau}\right) H_{n_{j}}$ converge to $\left(I-B_{\tau}\right) g$ weak* in $L^{\infty}(D)$. Since $\left(I-B_{\tau}\right) H_{n}=H_{n}-H_{n} * \tau$ converge to $h$ in norm, $h$ is the unique weak* limit of $\left(I-B_{\tau}\right) H_{n}$. Hence we have $h=\left(I-B_{\tau}\right) g=g-g * \tau \in A$. This completes the proof of the proposition.

\section{3. n-harmonicity}

Here we prove Theorem 1.4. Even though the theorem is true for every $n \in \mathbb{N}$, for the notational simplicity, in the proof we restrict ourselves to the case of $n=2$.

Proof of Theorem 1.4. Let $f \in L^{\infty}\left(D^{2}\right)$ and let us denote

$$
(T f)(z, w)=\iint_{D^{2}} f\left(\varphi_{z}(x), \varphi_{w}(y)\right) d \tau_{1}(x) d \tau_{2}(y)
$$

and then assume that $f$ satisfies $T f=f$.

First we prove the case that $f$ is 2-radial, i.e., $f(z, w)=f(|z|,|w|)$ for all $z, w \in D$.

Since $\tau_{1}(D)=\tau_{2}(D)=1$ for $v \in L_{R}^{\infty}(D)$, we can write by induction,

$\left(v * \tau_{1}^{n}\right)(z)=\int_{D} v(x) P_{n}(z, x) d \tau_{1}(x)$ and $\left(v * \tau_{2}^{n}\right)(z)=\int_{D} v(x) Q_{n}(z, x) d \tau_{2}(x)$ 
for some $P_{n}(z, x)$ and $Q_{n}(z, x)$ which satisfy

$$
\int_{D} P_{n}(z, x) d \tau_{1}(x)=\int_{D} Q_{n}(z, x) d \tau_{2}(x)=1 \text { for all } n \geq 1 .
$$

Since $\tau_{1}, \tau_{2}$ are power bounded, there is $M>0$ such that, for every $n \geq 1$,

$$
\left\|v * \tau_{1}^{n}\right\|_{\infty} \leq M\|v\|_{\infty} \text { and }\left\|v * \tau_{2}^{n}\right\|_{\infty} \leq M\|v\|_{\infty} .
$$

Now fix $w \in D$ and define $\left(f_{w}\right)_{n} \in L_{R}^{\infty}(D)$ as

$$
\left(f_{w}\right)_{n}(z)=\int_{D} f(z, y) Q_{n}(w, y) d \tau_{2}(y) .
$$

Then we get $\left\|\left(f_{w}\right)_{n}\right\|_{\infty} \leq M\|f\|_{\infty}$ for every $n \geq 1$. And we also get

$$
\begin{aligned}
\left(\left(f_{w}\right)_{n} * \tau_{1}^{n}\right)(z) & =\int_{D}\left(f_{w}\right)_{n}(x) P_{n}(z, x) d \tau_{1}(x) \\
& =\iint_{D^{2}} f(x, y) P_{n}(z, x) Q_{n}(z, y) d \tau_{1}(x) d \tau_{2}(y) \\
& =\left(T^{n} f\right)(z, w) .
\end{aligned}
$$

Now let $u \in L_{R}^{1}(\mu)$ satisfy $\int_{D} u d \mu=0$. Then for each fixed $w \in D$, we have

$$
\begin{aligned}
\int_{D} u(z) f(z, w) d \mu(z) & =\int_{D} u(z)\left(T^{n} f\right)(z, w) d \mu(z) \\
& =\int_{D} u(z)\left(\left(f_{w}\right)_{n} * \tau_{1}^{n}\right) d \mu(z) \\
& =\left(u *\left(f_{w}\right)_{n} * \tau_{1}^{n}\right)(0) \\
& =\left(\left(u * \tau_{1}^{n}\right) *\left(f_{w}\right)_{n}\right)(0) \\
& =\int_{D}\left(u * \tau_{1}^{n}\right)(z)\left(f_{w}\right)_{n}(z) d \mu(z)
\end{aligned}
$$

since the convolution is commutative and associative because $u, \tau,\left(f_{w}\right)_{n}$ are radial. Hence we get,

$$
\left|\int_{D} u(z) f(z, w) d \mu(z)\right| \leq\left\|\left(f_{w}\right)_{n}\right\|_{\infty}\left\|u * \tau_{1}^{n}\right\|_{L^{1}(\tau)} .
$$

Here we have $\left\|\left(f_{w}\right)_{n}\right\|_{\infty} \leq M\|f\|_{\infty}$ and by Theorem 1.1 ([3, Theorem 2.3]), we get

Hence we conclude that

$$
\left\|u * \tau_{1}^{n}\right\|_{L^{1}(\tau)} \rightarrow 0 \quad \text { as } \quad n \rightarrow \infty
$$

$$
\int_{D} u(z) f(z, w) d \mu(z)=0
$$

which means $f(z, w)$ is a constant for every fixed $w$; i.e., $f$ is a function of $w$ variable only.

Let $f(z, w)=g(w)$. Then $T f=f$ implies that $g * \tau_{2}=g$. Now applying Theorem 1.2 ([3, Theorem 3.1]), we conclude that $g$ is a constant (since $g$ is radial and harmonic). Hence $f$ is a constant. 
The rest of the proof follows a well-known traditional method (See, for example, proofs of Theorem 3.1 of [3] or Corollary 19 of [4]). Let $f \in L^{\infty}\left(D^{2}\right)$ satisfies $T f=f$. Then we consider the 2-radialization $R f$ of $f$ defined by

$$
(R f)(z, w)=\frac{1}{(2 \pi)^{2}} \int_{0}^{2 \pi} \int_{0}^{2 \pi} f\left(z e^{i \theta}, w e^{i \eta}\right) d \theta d \eta
$$

which satisfies, by Fubini's theorem, that $T(R f)=R(T f)=R f$. Hence by previous argument $R f$ is a constant, which means

$$
f(0,0)=\frac{1}{(2 \pi)^{2}} \int_{0}^{2 \pi} \int_{0}^{2 \pi} f\left(z e^{i \theta}, w e^{i \eta}\right) d \theta d \eta \quad \text { for all } z, w \in D .
$$

Now pick $(z, w) \in D^{2}$ and let $\psi \in \operatorname{Aut}\left(D^{2}\right)$ be defined by $\psi(x, y)=\left(\varphi_{z}(x)\right.$, $\left.\varphi_{w}(y)\right)$. Then by the rotation invariance of $\tau_{1}$ and $\tau_{2}$, we can easily get $T(f \circ$ $\psi)=T f \circ \psi=f \circ \psi$. Thus we can replace $f$ by $f \circ \psi$ in (3.1) to get

$$
f(z, w)=\frac{1}{(2 \pi)^{2}} \int_{0}^{2 \pi} \int_{0}^{2 \pi} f\left(\varphi_{z}\left(x e^{i \theta}\right), \varphi_{w}\left(y e^{i \eta}\right)\right) d \theta d \eta \text { for all } x, y \in D .
$$

Put $y=0$ in the above equation then use 4.2 .4 of [9] to get $\Delta_{1} f=0$, then we put $x=0$ to get $\Delta_{2} f=0$. Hence $f$ is 2-harmonic, which completes the proof of the theorem.

Acknowledgements. This research has been done while the author is visiting for a sabbatical year at the Korea Institute for Advanced Study. The author wants to express his heartfelt gratitude to the KIAS members for their hospitality during his visit.

\section{References}

[1] P. Ahern, M. Flores, and W. Rudin, An invariant volume-mean-value property, J. Funct. Anal. 111 (1993), no. 2, 380-397.

[2] J. Arazy and M. Engliš, Iterates and the boundary behavior of the Berezin transform, Ann. Inst. Fourier (Grenoble) 51 (2001), no. 4, 1101-1133.

[3] Y. Benyamini and Y. Weit, Harmonic analysis of spherical functions on SU(1,1), Ann. Inst. Fourier (Grenoble) 42 (1992), no. 3, 671-694.

[4] M. Engliš, Functions invariant under the Berezin transform, J. Funct. Anal. 121 (1994), no. $1,233-254$.

[5] H. Furstenberg, A Poisson formula for semi-simple Lie groups, Ann. of Math. (2) $\mathbf{7 7}$ (1963), 335-386.

[6] B Boundaries of Riemannian symmetric spaces, Symmetric spaces (Short Courses, Washington Univ., St. Louis, Mo., 1969-1970), pp. 359-377. Pure and Appl. Math., Vol. 8, Dekker, New York, 1972.

[7] Y. Katznelson and L. Tzafriri, On power bounded operators, J. Funct. Anal. 68 (1986), no. $3,313-328$

[8] J. Lee, Weighted Berezin transform in the polydisc, J. Math. Anal. Appl. 338 (2008), no. 2, 1489-1493.

[9] W. Rudin, Function Theory in the Unit Ball of $\mathbb{C}^{n}$, Springer-Verlag, New York Inc., 1980. 
Department of Mathematics

SOGANG UNIVERSITY

SEOUl 121-742, KoreA

E-mail address: jalee@sogang.ac.kr 